\title{
Investigation of the molecular mechanisms underlying myotonic dystrophy types 1 and 2 cataracts using microRNA-target gene networks
}

\author{
DEWANG SHAO ${ }^{1}$, XIAOQUAN ZHU ${ }^{1}$, WEI SUN ${ }^{1}$, LU HUO $^{1}$, \\ WEI CHEN ${ }^{2}$, HUA WANG ${ }^{2}$, BING LIU ${ }^{2}$ and PENG PAN ${ }^{1}$ \\ ${ }^{1}$ Department of Ophthalmology, Air Force Aviation Medicine Research Institute Affiliated Hospital; \\ ${ }^{2}$ Department of Ophthalmology, Air Force General Hospital, Beijing 100089, P.R. China
}

Received February 26, 2016; Accepted February 23, 2017

DOI: $10.3892 / \mathrm{mmr} .2017 .7059$

\begin{abstract}
The purpose of the present study was to investigate the molecular mechanisms of myotonic dystrophy (DM) 1 and 2 cataracts using bioinformatics methods. A microarray dataset (E-MEXP-3365) downloaded from the Array Express database included lens epithelial samples of DM1 and DM2 cataract patients ( $\mathrm{n}=3 /$ group) and non-DM lens epithelial samples as a control $(n=4)$. Differentially expressed genes (DEGs) were identified between DM1 and control samples, and between DM2 and control samples. Pathway enrichment analyses were performed for the DEGs. Potential micro (mi)RNAs regulating these DEGs were predicted. An miRNA-target gene network was constructed for DM1 and DM2. The study identified 223 DEGs in DM1, and 303 DEGs in DM2. DM1 and DM2 shared 172 DEGs. The DEGs in DM1 were enriched with calcium, Wnt and axon guidance signaling pathways. The DEGs in DM2 were linked by adherens junction signaling pathways. miRNA (miR)-197, miR-29b and miR-29c were included in the network modules of DM1. miR-197, miR-29c and miR-29a were involved in the network modules of DM2. It is therefore hypothesized that these signaling pathways and miRNAs underlie DM1 and DM2 cataracts, and may represent potential therapeutic targets for the treatment of this disorder.
\end{abstract}

\section{Introduction}

Myotonic dystrophy (DM) is a progressive multi-systemic disease consisting of highly heterogeneous clinical syndromes, affecting $\sim 1$ in 8,000 people globally (1). It has two subtypes:

Correspondence to: Dr Dewang Shao, Department of Ophthalmology, Air Force Aviation Medicine Research Institute Affiliated Hospital, 15 Changyungong, Haidian, Beijing 100089, P.R. China

E-mail: shaodengwei@hotmail.com

Key words: microRNA, pathway, gene ontology, Kyoto Encyclopedia of Genes and Genomes, cataract
DM1 and DM2. DM1 is primarily attributed to the mutation of a CTG expansion residing in the 3'-untranslated region of the dystrophia myotonica-protein kinase (DMPK) gene, whereas DM2 is ascribed to an untranslated CCTG expansion in the cellular retroviral nucleic acid binding protein 1/zinc finger 9 (CNBP) gene (2). Several body components are affected by the disease, including skeletal muscles, the central nervous system, the heart and the eyes (3). DM causes various ocular syndromes, such as early onset cataracts, external ophthalmoplegia and pigmentary retinopathy $(4,5)$.

DM1 and DM2 share many clinical features, and their underlying mechanisms are often investigated jointly. It has been reported that the stability of mutant RNAs (CUG and CCUG) in DM1 and DM2 is increased as a consequence of deficiency of RNA helicase p68 (6). Dysregulation of intracellular calcium homeostasis in DM1 and DM2 myotubes has been identified to be associated with the aberrant splicing of $\mathrm{Ca}^{2+}$ handling genes (7). Bachinski et al (8) suggested that DM1 and DM2 are largely identical, as they are associated with dysregulated expression of genes in skeletal muscles. Previous studies have yielded significant findings concerning the molecular mechanisms of DM1 and DM2 $(9,10)$. However, little attention has been paid to the molecular mechanisms of DM1 and DM2 cataracts. DM1 and DM2 cataracts are almost identical in appearance and age of onset $(11,12)$. Rhodes et al (13) demonstrated that upregulated interferon-regulated genes and the type 1 interferon signaling pathway may be involved in the common mechanism of DM1 and DM2 cataracts. Further efforts are required to unveil the underlying molecular mechanisms.

With the aid of high output microarray analysis, the present study identified differentially expressed genes (DEGs) between DM1 and non-DM cataracts samples, and between DM2 and non-DM cataracts samples. Gene ontology (GO) and the Kyoto Encyclopedia of Genes and Genomes (KEGG) pathway enrichment analysis were performed to investigate the biological functions and pathways that may involve the DEGs. Furthermore, the present study examined potential micro (mi)RNAs that may be involved in DM1 and DM2 by constructing a microRNA (miRNA)-gene network for DM1 and DM2, respectively. 


\section{Materials and methods}

Microarray dataset. The present study is a secondary study using the microarray dataset (E-MEXP-3365) (13) downloaded from the Array Express database (http://www.ebi .ac.uk/arrayexpress) (14), including 3 lens epithelial samples from DM1 cataract patients, 3 lens epithelial samples from DM2 cataract patients and 4 non-DM lens epithelial samples (control).

Data preprocessing and identification of DEGs. The microarray data were preprocessed using the lumi Bioconductor package version $2.3(15,16)$. Each probe was mapped to its corresponding gene, followed by background correction and quartile data normalization. If a probe corresponded to $>1$ gene, the probe would be deleted. When several probes mapped to one gene, the mean expression values of these probes were calculated as the expression value of the gene.

Differentially expressed genes were identified between DM1 cataract samples and non-DM cataract samples, and between DM2 cataract and non-DM cataract samples, using a t-test. The strict cutoff was set at fold change $(\mid \log F C l)>2$ and false discovery rate $(\mathrm{FDR})<0.05$.

GO and KEGG pathway enrichment analysis. In order to investigate the biological function involving the DEGs identified in the DM1 and DM2 cataracts, GO (17) and KEGG (18) pathway enrichment analysis was performed using Database for Annotation, Visualization and Integrated Discovery (https://david.ncifcrf.gov/tools.jsp) (19). Significant GO terms and KEGG pathways (adjusted $\mathrm{P} \leq 0.05$, gene number $\geq 2$ ) were identified. There were 3 types of GO terms: Biological process (BP), cellular component (CC) and molecular function (MF).

Construction of miRNA-DEG networks and analysis of network modules. Potential miRNAs that may be involved in regulating the DEGs identified in MD1 and MD2 cataract $\mathrm{S}$ were predicted using 7 algorithms, including PicTar (20), DIANA-microT (21), miRanda (22), miRBase (23), RNAhybrid (24), RNA22 (25) and TargetScan (26). Only the miRNAs that were validated using at least 3 out of the 7 algorithms were selected. With the predicted miRNAs and DEGs, a miRNA-gene network was constructed for DM1 and DM2, respectively, using Cytoscape software version 3.2.0 (27). In the network, a node denoted a gene or a miRNA; an undirected link represented an interaction between two genes, or between a gene and a miRNA. The degree of a node referred to the number of interactions of a gene or a miRNA. Furthermore, significant network modules were extracted from the network using Mcode plugin in Cytoscape (27) (cutoff value: Degree 22) and analyzed.

\section{Results}

Identification of DEGs. A total of 223 DEGs were screened between DM1 cataract and non-DM cataract samples. A total of 303 DEGs were screened between DM2 cataract and non-MD cataract samples. Furthermore, 172 DEGs were shared by DM1 and DM2 cataracts.
GO and KEGG pathway enrichment analysis. GO enrichment analysis revealed that most of significant $\mathrm{CC}$ and MF terms were common to DM1 and DM2 cataracts (Tables I and II). The overlapped MF terms were related to nucleotide binding and ribonucleotide binding. With regards to BP terms, DEGs in DM1 cataracts were significantly enriched with intracellular transport, cell cycle, mRNA metabolic process, RNA processing and vesicle-mediated transport (Table I), whereas the DEGs in DM2 cataract were significantly associated with regulation of apoptosis, regulation of programmed cell death, regulation of cell death, intracellular transport and vesicle-mediated transport (Table II).

The KEGG pathway enrichment analysis revealed that the amyotrophic lateral sclerosis and Alzheimer's disease signaling pathways were shared by DM1 and D2 cataracts. Furthermore, DEGs in DM1 cataracts were significantly enriched with calcium signaling, and markers from the Wnt and axon guidance signaling pathways (Table III), whereas DEGs in DM2 cataracts were markedly associated with the adherens junction signaling pathway (Table IV).

Analysis of miRNA-gene networks. In the current study, a total of 345 miRNAs were predicted to be associated with the DEGs identified in DM1 cataracts, which constituted a total of 1,022 miRNA-target gene pairs. The DEGs in DM1 cataracts were predicted to be regulated by 371 miRNAs, and a total of 1,357 miRNA-target gene pairs were obtained.

For the purpose of deciphering the associations between DEGs, and between DEGs and the predicted miRNAs, an miRNA-gene network was constructed for DM1 (Fig. 1) and DM2 (Fig. 2). A total of three modules were extracted from the miRNA-gene network for DM1 cataracts. As presented in Fig. 3, miR-197, miR-29b, miR-29c, miR-130a and miR-130b were included in the network modules. A total of seven modules were extracted from the miRNA-gene network for DM2 cataracts (Fig. 4). miR-197, miR-29c, miR-29c, miR-152, miR-302d, miR-302d, miR-372, miR-20a, miR-20b, miR-133a, miR-133b, miR-148a, miR-148b and miR-515-3p were involved in these network modules. miR-197 and miR-29c were shared by the network modules of DM1 and DM2.

\section{Discussion}

There are limited studies specifically focused on the molecular mechanisms of DM1 and DM2 cataracts. The present study identified 223 DEGs in DM1 cataracts and 303 DEGs in DM2 cataracts. Notably, a total of 172 overlapped DEGs were identified in DM1 and in DM2 cataracts. They shared several identical significant GO terms, but there were additionally some GO terms specific to DM1 or DM2 cataracts. DEGs in DM1 cataracts were significantly enriched in the calcium, Wnt and axon guidance signaling pathways, whereas DEGs in DM2 cataracts were significantly associated with the adherens junction signaling pathway. One highlight of the current study was the construction of an miRNA-gene network for DM1 and DM2 cataracts. miR-197, miR-29b, miR-29c, miR-130a and miR-130b were included in the three network modules extracted from the miRNA-gene network for DM1 cataracts. miR-197, miR-29c, miR-29c, miR-152, miR-302d, miR-372, miR-20a, miR-20b, miR-133a, miR-133b, miR-148a, miR-148b 
Table I. Significant GO terms for DEGs in DM1 cataracts.

\begin{tabular}{|c|c|c|c|}
\hline GO terms & Description & Count of genes & Q-value \\
\hline \multirow[t]{10}{*}{$\mathrm{BP}$} & Intracellular transport & 18 & 0.011184278 \\
\hline & Cell cycle & 18 & 0.045910811 \\
\hline & mRNA metabolic process & 15 & $7.41 \mathrm{E}-04$ \\
\hline & RNA processing & 15 & 0.022266862 \\
\hline & Vesicle-mediated transport & 15 & 0.03260547 \\
\hline & mRNA processing & 14 & $6.10 \mathrm{E}-04$ \\
\hline & Protein folding & 13 & $7.45 \mathrm{E}-06$ \\
\hline & Cytoskeleton organization & 13 & 0.020266359 \\
\hline & Regulation of cellular protein metabolic process & 13 & 0.035471916 \\
\hline & RNA splicing & 12 & 0.002271744 \\
\hline \multirow[t]{10}{*}{$\mathrm{CC}$} & Non-membrane-bounded organelle & 51 & 0.00607 \\
\hline & Intracellular non-membrane-bounded organelle & 51 & 0.00607 \\
\hline & Membrane-enclosed lumen & 48 & $1.60 \mathrm{E}-05$ \\
\hline & Organelle lumen & 46 & 4.69E-05 \\
\hline & Intracellular organelle lumen & 45 & $5.82 \mathrm{E}-05$ \\
\hline & Cytosol & 32 & 0.002344 \\
\hline & Nuclear lumen & 32 & 0.008374 \\
\hline & Vesicle & 22 & $3.57 \mathrm{E}-04$ \\
\hline & Membrane-bounded vesicle & 21 & $1.08 \mathrm{E}-04$ \\
\hline & Cytoplasmic vesicle & 21 & $5.36 \mathrm{E}-04$ \\
\hline \multirow[t]{10}{*}{ MF } & Nucleotide binding & 39 & 0.075474 \\
\hline & Purine ribonucleotide binding & 34 & 0.049174 \\
\hline & Ribonucleotide binding & 34 & 0.049174 \\
\hline & Purine nucleotide binding & 34 & 0.08014 \\
\hline & Nucleoside binding & 30 & 0.063175 \\
\hline & ATP binding & 29 & 0.039048 \\
\hline & Adenyl ribonucleotide binding & 29 & 0.045135 \\
\hline & Adenyl nucleotide binding & 29 & 0.076671 \\
\hline & Purine nucleoside binding & 29 & 0.088577 \\
\hline & RNA binding & 20 & 0.003752 \\
\hline
\end{tabular}

GO, gene ontology; DEG, differentially expressed genes; DM, myotonic dystrophy; BP, biological process; CC, cellular component; MF, molecular function.

and miR-515-3p were involved in the seven network modules extracted from the miRNA-gene network for DM2 cataract. Notably, miR-197 and miR-29c were shared between DM1 and DM2 cataracts.

It has been demonstrated that DM1 and DM2 share several clinical and genetic features (2). Both of them are toxic RNA diseases. Rhodes et al (13) identified a high proportion of overlapped dysregulated genes in DM1 and DM2 cataracts, and suggested the involvement of the innate immune response and interferon signaling in their common underlying mechanisms. The present study demonstrated that DM1 and DM2 cataracts shared 172 DEGs. Furthermore, the two forms of $\mathrm{DM}$ cataracts were associated with identical CC and MF terms associated with nucleotide binding and ribonucleotide binding in the present study. In addition, they were associated intracellular transport and vesicle-mediated transport. These findings indicated that nucleotide binding, ribonucleotide binding, intracellular transport and vesicle-mediated transport may be implicated in the common molecular mechanisms underlying DM1 and DM2 cataracts.

DM1 and DM2 are two genetically distinct entities. DM1 is due to a mutation of the CTG expansion in DMPK, whereas DM2 is caused by a CCTG expansion in the CNBP gene (9). DM2 is rarer and has a milder phenotype and later onset of symptoms, when compared with DM1 (28). The DM1 repeat number increases over the generations. Conversely, the $\mathrm{DM} 2$ repeat number may decrease over the generations (12). In addition to the common molecular mechanisms, differences do exist between DM1 and DM2 (29). Franc et al (30) demonstrated that the gray matter volumes are significantly decreased in DM1 patients, rather than in DM2 patients. Passeri et al (31) identified that the phosphate levels are decreased in DM1 patients, but not in DM2 patients. In the present study, results of GO functional enrichment analysis indicated that the DEGs in DM1 cataracts may affect cell cycle, mRNA metabolic process and RNA processing, whereas 
Table II. Significant GO terms for DEGs in DM2 cataracts.

\begin{tabular}{|c|c|c|c|}
\hline GO term & Description & Gene count & Q-value \\
\hline \multirow[t]{10}{*}{$\mathrm{BP}$} & Regulation of apoptosis & 25 & 0.015950693 \\
\hline & Regulation of programmed cell death & 25 & 0.017739841 \\
\hline & Regulation of cell death & 25 & 0.018408079 \\
\hline & Protein transport & 23 & 0.028778834 \\
\hline & Establishment of protein localization & 23 & 0.031394209 \\
\hline & Intracellular transport & 22 & 0.011776008 \\
\hline & Vesicle-mediated transport & 19 & 0.023259474 \\
\hline & Membrane organization & 18 & 8.39E-04 \\
\hline & Protein complex assembly & 17 & 0.028082861 \\
\hline & Protein complex biogenesis & 17 & 0.028082861 \\
\hline \multirow[t]{10}{*}{$\mathrm{CC}$} & Intracellular non-membrane-bounded Organelle & 59 & 0.039736 \\
\hline & Non-membrane-bounded organelle & 59 & 0.039736 \\
\hline & Membrane-enclosed lumen & 57 & 4.79E-05 \\
\hline & Organelle lumen & 53 & $3.82 \mathrm{E}-04$ \\
\hline & Intracellular organelle lumen & 52 & 4.07E-04 \\
\hline & Cytosol & 48 & 4.18E-06 \\
\hline & Nuclear lumen & 38 & 0.017793 \\
\hline & Organelle membrane & 34 & 0.002298 \\
\hline & Mitochondrion & 30 & 0.020663 \\
\hline & Endomembrane system & 29 & $3.82 \mathrm{E}-04$ \\
\hline \multirow[t]{10}{*}{$\mathrm{MF}$} & Nucleotide binding & 53 & 0.037027 \\
\hline & Purine nucleotide binding & 44 & 0.085847 \\
\hline & Ribonucleotide binding & 42 & 0.096712 \\
\hline & Purine ribonucleotide binding & 42 & 0.096712 \\
\hline & Nucleoside binding & 39 & 0.057925 \\
\hline & Adenyl nucleotide binding & 38 & 0.064446 \\
\hline & Purine nucleoside binding & 38 & 0.076716 \\
\hline & ATP binding & 36 & 0.063612 \\
\hline & Adenyl ribonucleotide binding & 36 & 0.073955 \\
\hline & RNA binding & 26 & 0.001454 \\
\hline
\end{tabular}

KEGG, Kyoto Encyclopedia of Genes and Genomes; DEG, differentially expressed genes; DM, myotonic dystrophy; BP, biological process; $\mathrm{CC}$, cellular component; MF, molecular function; ATP, adenosine triphosphate.

Table III. KEGG pathways for DEGs in DM1 cataracts.

\begin{tabular}{lcll}
\hline KEGG & Gene count & Adjusted P-value & \multicolumn{1}{c}{ Description } \\
\hline KEGG_PATHWAY & 5 & 0.020744 & Long-term potentiation \\
KEGG_PATHWAY & 4 & 0.048553 & Amyotrophic lateral sclerosis \\
KEGG_PATHWAY & 6 & 0.10831 & Alzheimer's disease \\
KEGG_PATHWAY & 5 & 0.131481 & Spliceosome \\
KEGG_PATHWAY & 6 & 0.137285 & Calcium signaling pathway \\
KEGG_PATHWAY & 4 & 0.171196 & Dilated cardiomyopathy \\
KEGG_PATHWAY & 5 & 0.206866 & Wnt signaling pathway \\
KEGG_PATHWAY & 3 & 0.221622 & Pathogenic Escherichia coli infection \\
KEGG_PATHWAY & 4 & 0.243621 & Oocyte meiosis \\
KEGG_PATHWAY & 4 & 0.32439 & Axon guidance \\
\hline
\end{tabular}

KEGG, Kyoto Encyclopedia of Genes and Genomes; DEG, differentially expressed genes; DM, myotonic dystrophy. 
Table IV. KEGG pathways for DEGs in DM2 cataracts.

\begin{tabular}{lcll}
\hline KEGG & Gene count & Q-value & \multicolumn{1}{c}{ Description } \\
\hline KEGG_PATHWAY & 10 & 0.013726 & Huntington's disease \\
KEGG_PATHWAY & 4 & 0.037286 & Basal transcription factors \\
KEGG_PATHWAY & 7 & 0.053893 & Parkinson's disease \\
KEGG_PATHWAY & 5 & 0.080184 & Adherens junction \\
KEGG_PATHWAY & 4 & 0.101893 & Amyotrophic lateral sclerosis \\
KEGG_PATHWAY & 4 & 0.106299 & Non-small cell lung cancer \\
KEGG_PATHWAY & 4 & 0.119952 & Pathogenic Escherichia coli infection \\
KEGG_PATHWAY & 2 & 0.120912 & Methane metabolism \\
KEGG_PATHWAY & 7 & 0.131916 & Alzheimer's disease \\
KEGG_PATHWAY & 4 & 0.14904 & Glioma
\end{tabular}

KEGG, Kyoto Encyclopedia of Genes and Genomes; DEG, differentially expressed genes; DM, myotonic dystrophy.

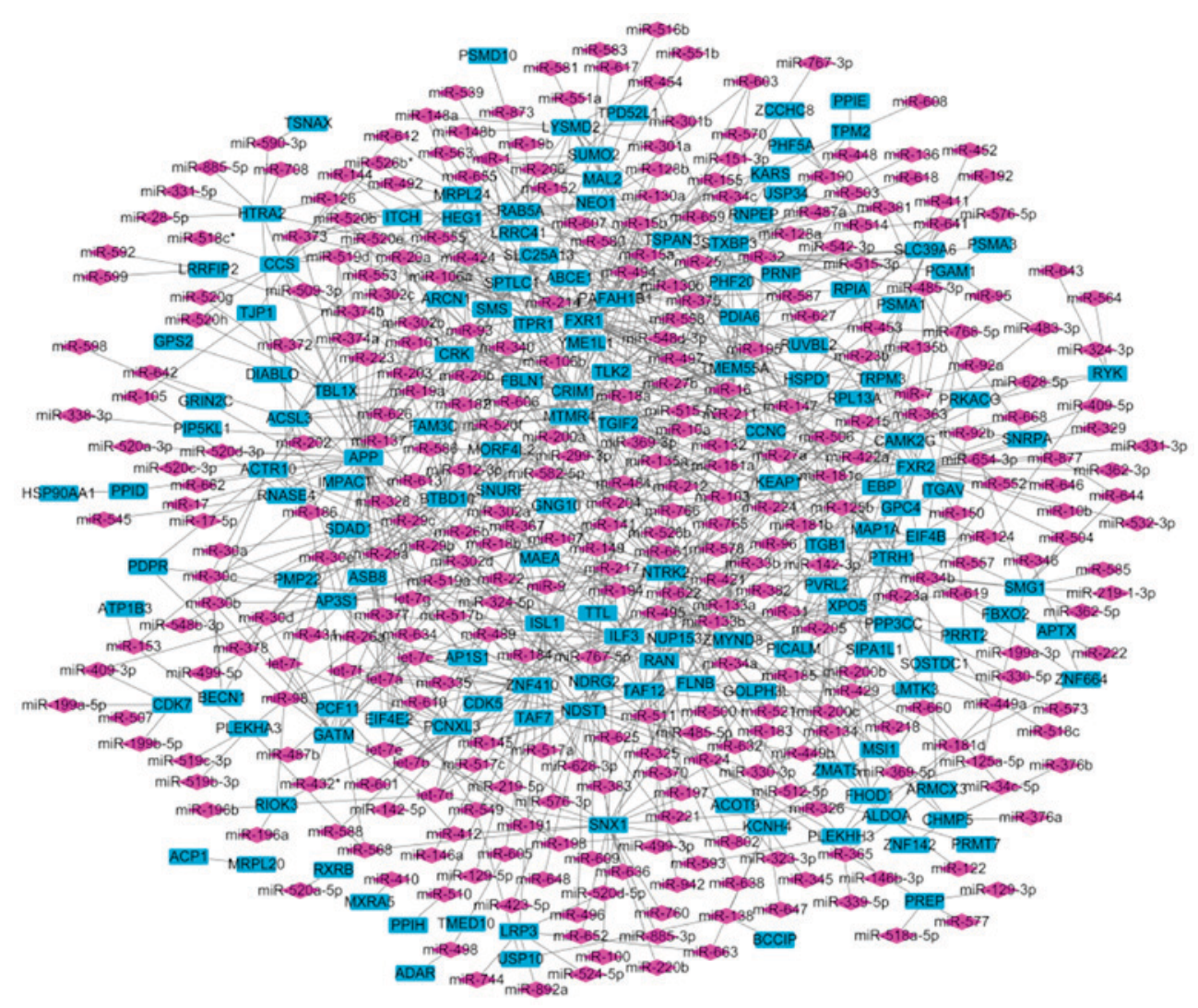

Figure 1. An miRNA-gene network for DM1 cataracts. Square nodes denote genes and diamond-shaped nodes denote miRNAs. An undirected link represents an interaction between two genes, or between a gene and an miRNA. miRNA, microRNA; DM, myotonic dystrophy.

the DEGs in DM2 cataract may be involved in regulation of apoptosis, regulation of programmed cell death and regulation of cell death. These results provide evidences in favor of the discrepancy in the underlying molecular mechanisms between DM1 and DM2 cataracts.

It has been presented that, in DM1, unstable CTG repeats in the DMPK gene induce mis-splicing to fetal/neonatal isoforms of many transcripts associated with cellular $\mathrm{Ca} 2+$ homeostasis, and several calcium-associated proteins are abnormally expressed in DM1 and DM2, including $\mathrm{Ca}^{2+}$ release channel ryanodine receptor and calsequestrin 2 (32). Botta et al (33) suggested that unbalanced $\mathrm{Ca}^{2+}$ homeostasis causes muscle degeneration in DM1 muscle cells by activating endoplasmic reticulum stress. In support of these findings, the present study indicated that DEGs in DM1 cataracts were significantly enriched in the calcium signaling pathway, indicating that this may serve a role in the pathogenesis of DM1 cataracts. Furthermore, results of the KEGG pathway enrichment analysis suggested that the Wnt axon guidance signaling pathways may be involved in DM1 cataract, whereas 


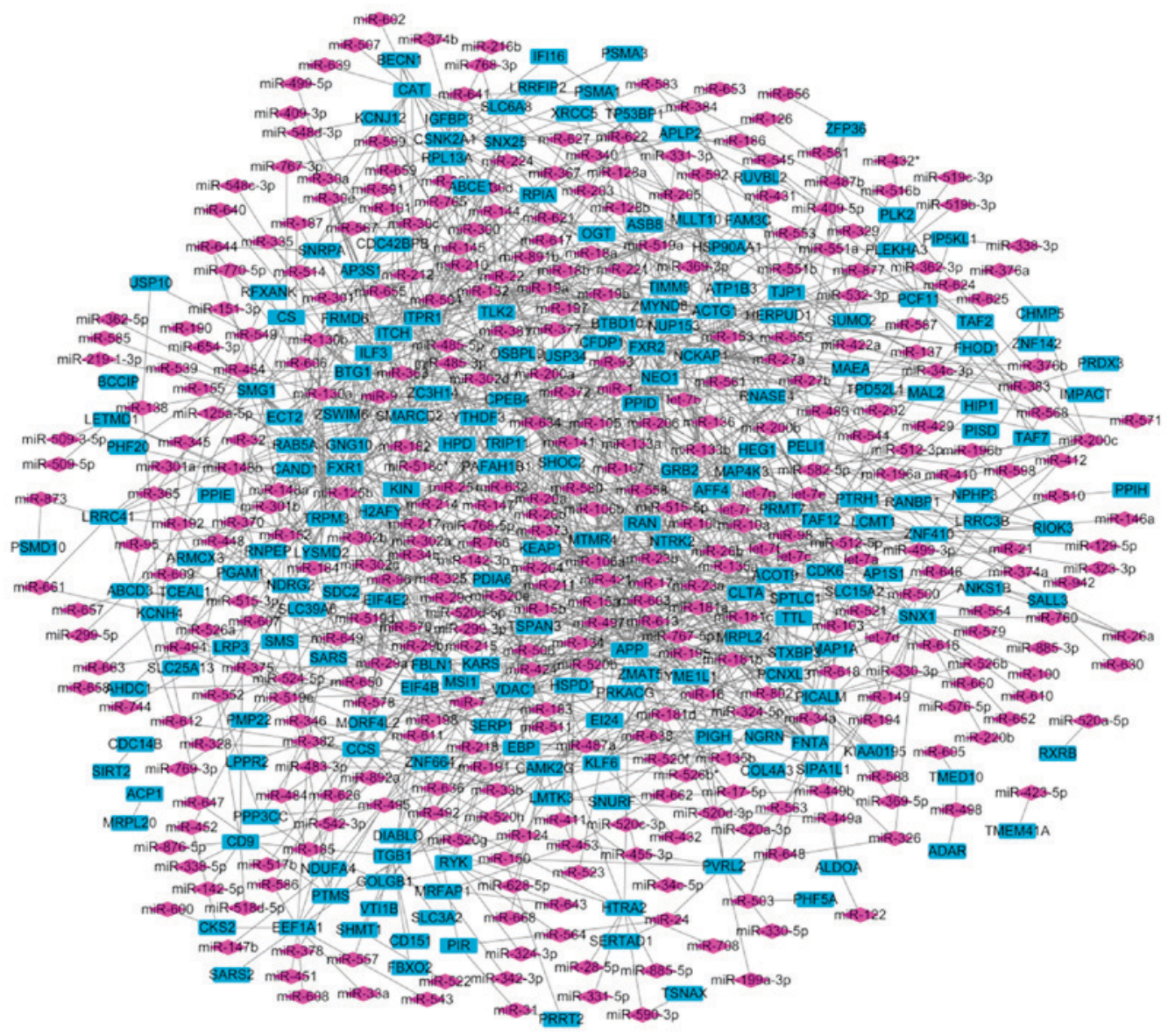

Figure 2. miRNA-gene network for DM2 cataracts. Square nodes denote genes and diamond-shaped nodes denote miRNAs. An undirected link represents an interaction between two genes, or between a gene and an miRNA. miRNA, microRNA; DM, myotonic dystrophy.

A

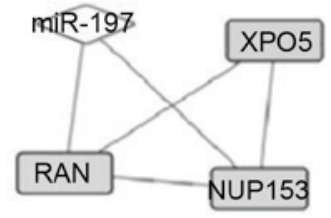

B

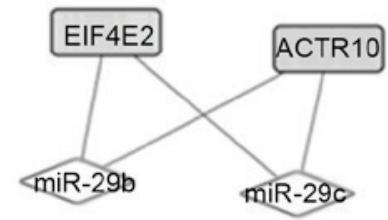

C

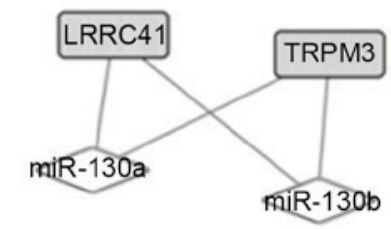

Figure 3. (A-C) A total of three modules extracted from the miRNA-gene network for DM1 cataract. Square nodes denote genes and diamond-shaped nodes denote miRNAs. An undirected link stands for an interaction between two genes, or between a gene and an miRNA. miRNA, microRNA; DM, myotonic dystrophy.

A

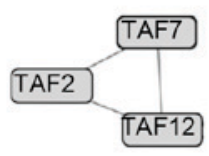

E

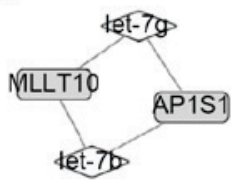

B

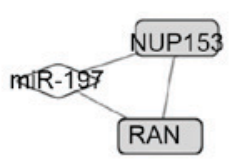

F

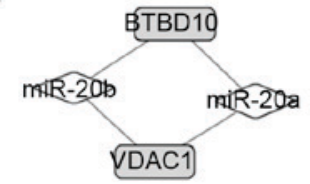

C

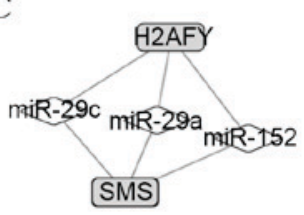

D<smiles>CCC1CCCC1[18O][Ba]</smiles>

$$
\begin{aligned}
& \text { G CLTA } \\
& \text { miR-133b miR-133a } \\
& \text { ZC3H14 } \\
& \text { miR-148a miR-148b } \\
& \text { LRRC41 ABCD3 }
\end{aligned}
$$

Figure 4. (A-G) A total of seven modules extracted from the miRNA-gene network for DM2 cataracts. Square nodes represent genes and diamond-shaped nodes represent miRNAs. An undirected link refers to an interaction between two genes, or between a gene and an miRNA. 
the adherens junction signaling pathway may be involved in DM2 cataracts. To the best of our knowledge, these findings have not been reported before.

miRNAs are highly conserved, noncoding RNAs that are involved in a wide range of biological processes, including myogenesis and muscle regeneration. The deregulation of miRNAs in the pathogenesis of MD has been an area of intense investigation. Perbellini et al (34) discovered that miR-29b, miR-29c and miR-33 are downregulated in skeletal muscle biopsies of DM1 patients relative to controls. Greco et al (35) created a list of dysregulated miRNAs in skeletal muscle biopsies of DM2 patients compared with controls, including miR-34a-5p, miR-34b-3p and miR-34c-5p. Furthermore, miR-206 is overexpressed in the skeletal muscle of DM1 patients (36), and miR-1 is associated with heart defects in DM1 and DM2 (37). However, the dysregulated miRNAs in the pathogenesis of DM1 and DM2 cataracts remain to be clearly defined. The present study suggested that miR-197, miR-29b, miR-29c, miR-130a and miR-130b are potentially involved in DM1 cataracts, while miR-197, miR-29c, miR-29a, miR-152, miR-302d, miR-372, miR-20a, miR-20b, miR-133a, miR-133b, miR-148a, miR-148b and miR-515-3p may be implicated in DM2 cataracts. miR-197 and miR-29c were involved in DM1 and DM2 cataracts. The present study added more insights concerning miRNA deregulation in the pathogenesis of DM.

The results of the current study advanced understanding of the molecular mechanisms underlying DM1 or DM2 cataracts. However, it had a limited sample size. More importantly, further experimental studies are warranted to verify these findings derived from the microarray data analysis.

In conclusion, the present study identified potential genes, pathways and upstream miRNAs, which may be involved in the pathogenesis of DM1 or DM2 cataracts. The calcium, Wnt and axon guidance signaling pathways, and miR-197, miR-29b and miR-29c, may be involved in DM1 cataracts. The adherens junction signaling pathway, miR-197, miR-29c and miR-29a may be involved in DM2 cataracts. Further studies are required to elucidate the different underlying molecular mechanisms between DM1 and DM2 cataracts, and to develop potential treatments for this disorder.

\section{References}

1. Thornton CA: Myotonic dystrophy. Neurol Clin 32: 705-719, 2014.

2. Ranum LP and Day JW: Myotonic dystrophy: Clinical and molecular parallels between myotonic dystrophy type 1 and type 2. Curr Neurol Neurosci Rep 2: 465-470, 2002

3. Kumar A, Agarwal S, Agarwal D and Phadke SR: Myotonic dystrophy type 1 (DM1): A triplet repeat expansion disorder. Gene 522: 226-230, 2013.

4. Lessell S, Coppeto J and Samet S: Ophthalmoplegia in myotonic dystrophy. Am J Ophthalmol 71: 1231-1235, 1971.

5. Burian HM and Burns CA: Ocular changes in myotonic dystrophy. Am J Ophthalmol 63: 22-34, 1967.

6. Jones K, Wei C, Schoser B, Meola G, Timchenko N and Timchenko L: Reduction of toxic RNAs in myotonic dystrophies type 1 and type 2 by the RNA helicase p68/DDX5. Proc Natl Acad Sci USA 112: 8041-8045, 2015.

7. Santoro M, Piacentini R, Masciullo M, Bianchi ML, Modoni A, Podda MV, Ricci E, Silvestri G and Grassi C: Alternative splicing alterations of $\mathrm{Ca} 2+$ handling genes are associated with $\mathrm{Ca} 2+$ signal dysregulation in myotonic dystrophy type 1 (DM1) and type 2 (DM2) myotubes. Neuropathol Appl Neurobiol 40: 464-476, 2013.
8. Bachinski LL, Baggerly KA, Neubauer VL, Nixon TJ, Raheem O, Sirito M, Unruh AK, Zhang J, Nagarajan L, Timchenko LT, et al: Most expression and splicing changes in myotonic dystrophy type 1 and type 2 skeletal muscle are shared with other muscular dystrophies. Neuromuscul Disord 24: 227-240, 2014.

9. Liquori CL, Ricker K, Moseley ML, Jacobsen JF, Kress W, Naylor SL, Day JW and Ranum LP: Myotonic dystrophy type 2 caused by a CCTG expansion in intron 1 of ZNF9. Science 293: 864-867, 2001

10. Hino SI, Kondo S, Sekiya H, Saito A, Kanemoto S, Murakami T, Chihara K, Aoki Y, Nakamori M, Takahashi MP and Imaizumi K: Molecular mechanisms responsible for aberrant splicing of SERCA1 in myotonic dystrophy type 1. Hum Mol Genet 16: 2834-2843, 2007.

11. Spiro AJ: Myotonic dystrophy, 3rd edition. Pediatric Neurology, 27: 76, 2002.

12. Day J, Ricker K, Jacobsen JF, Rasmussen LJ, Dick KA, Kress W, Schneider C, Koch MC, Beilman GJ, Harrison AR, et al: Myotonic dystrophy type 2: Molecular, diagnostic and clinical spectrum. Neurology 60: 657-664, 2003.

13. Rhodes JD, Lott MC, Russell SL, Moulton V, Sanderson J, Wormstone IM and Broadway DC: Activation of the innate immune response and interferon signalling in myotonic dystrophy type 1 and type 2 cataracts. Hum Mol Genet 21: 852-862, 2012.

14. Parkinson H, Kapushesky M, Shojatalab M, Abeygunawardena N, Coulson R, Farne A, Holloway E, Kolesnykov N, Lilja P, Lukk M, et al: ArrayExpress-a public database of microarray experiments and gene expression profiles. Nucleic Acids Res 35 (Database issue): D747-D750, 2007.

15. Du P, Kibbe WA and Lin SM: lumi: A pipeline for processing Illumina microarray. Bioinformatics 24: 1547-1548, 2008.

16. Gentleman RC, Carey VJ, Bates DM, Bolstad B, Dettling M, Dudoit S, Ellis B, Gautier L, Ge Y, Gentry J, et al: Bioconductor: Open software development for computational biology and bioinformatics. Genome Biol 5: R80, 2004.

17. Harris MA, Clark J, Ireland A, Lomax J, Ashburner M, Foulger R, Eilbeck K, Lewis S, Marshall B, Mungall C, et al: The gene ontology (GO) database and informatics resource. Nucleic Acids Res 32 (Database issue): D258-D261, 2004.

18. Kanehisa M and Goto S: KEGG: Kyoto encyclopedia of genes and genomes. Nucleic Acids Res 28: 27-30, 2000.

19. Dennis G Jr, Sherman BT, Hosack DA, Yang J, Gao W, Lane HC and Lempicki RA: DAVID: Database for annotation, visualization and integrated discovery. Genome biol 4: P3, 2003.

20. Krek A, Grün D, Poy MN, Wolf R, Rosenberg L, Epstein EJ, MacMenamin P, da Piedade I, Gunsalus KC, Stoffel M and Rajewsky N: Combinatorial microRNA target predictions. Nat Genet 37: 495-500, 2005.

21. Maragkakis M, Vergoulis T, Alexiou P, Reczko M, Plomaritou K, Gousis M, Kourtis K, Koziris N, Dalamagas T and Hatzigeorgiou AG: DIANA-microT Web server upgrade supports Fly and Worm miRNA target prediction and bibliographic miRNA to disease association. Nucleic Acids Res 39 (Web Server issue): W145-W148, 2011

22. Enright AJ, John B, Gaul U, Tuschl T, Sander C and Marks DS: MicroRNA targets in Drosophila. Genome Biol 5: R1, 2003.

23. Griffiths-Jones S, Grocock RJ, van Dongen S, Bateman A and Enright AJ: miRBase: MicroRNA sequences, targets and gene nomenclature. Nucleic Acids Res 34 (Database issue): D140-D144, 2006.

24. Kruger J and Rehmsmeier M: RNAhybrid: microRNA target prediction easy, fast and flexible. Nucleic Acids Res 34 (Web Server issue): W451-W454, 2006.

25. Loher P and Rigoutsos I: Interactive exploration of RNA22 microRNA target predictions. Bioinformatics 28: 3322-3323, 2012.

26. Lewis BP, Burge CB and Bartel DP: Conserved seed pairing, often flanked by adenosines, indicates that thousands of human genes are microRNA targets. Cell 120: 15-20, 2005.

27. Kohl M, Wiese S and Warscheid B: Cytoscape: software for visualization and analysis of biological networks. Methods Mol Biol 696: 291-303, 2011.

28. Turner C and Hilton-Jones D: The myotonic dystrophies: Diagnosis and management. J Neurol Neurosurg Psychiatry 81: 358-367, 2010.

29. Ulane CM, Teed S and Sampson J: Recent advances in myotonic dystrophy type 2. Curr Neurol Neurosci Rep 14: 429, 2014.

30. Franc DT, Muetzel RL, Robinson PR, Rodriguez CP, Dalton JC, Naughton CE, Mueller BA, Wozniak JR, Lim KO and Day JW: Cerebral and muscle MRI abnormalities in myotonic dystrophy. Neuromuscul Disord 22: 483-491, 2012. 
31. Passeri E, Bugiardini E, Sansone VA, Valaperta R, Costa E, Ambrosi B, Meola G and Corbetta S: Vitamin D, parathyroid hormone and muscle impairment in myotonic dystrophies. J Neurol Sci 331: 132-135, 2013

32. Vihola A, Sirito M, Bachinski LL, Raheem O, Screen M, Suominen T, Krahe R and Udd B: Altered expression and splicing of $\mathrm{Ca}(2+)$ metabolism genes in myotonic dystrophies DM1 and DM2. Neuropathol Appl Neurobiol 39: 390-405, 2013.

33. Botta A, Malena A, Loro E, Del Moro G, Suman M, Pantic B, Szabadkai G and Vergani L: Altered Ca2+ homeostasis and endoplasmic reticulum stress in myotonic dystrophy type 1 muscle cells. Genes (Basel) 4: 275-292, 2013.

34. Perbellini R, Greco S, Sarra-Ferraris G, Cardani R, Capogrossi MC, Meola G and Martelli F: Dysregulation and cellular mislocalization of specific miRNAs in myotonic dystrophy type 1. Neuromuscul Disord 21: 81-88, 2011.
35. Greco S, Perfetti A, Fasanaro P, Cardani R, Capogrossi MC, Meola G and Martelli F: Deregulated microRNAs in myotonic dystrophy type 2. PLos One 7: e39732, 2012.

36. Gambardella S, Rinaldi F, Lepore SM, Viola A, Loro E, Angelini C, Vergani L, Novelli G and Botta A: Overexpression of microRNA-206 in the skeletal muscle from myotonic dystrophy type 1 patients. J Transl Med 8: 48, 2010.

37. Rau F, Freyermuth F, Fugier C, Villemin JP, Fischer MC, Jost B, Dembele D, Gourdon G, Nicole A, Duboc D, et al: Misregulation of miR-1 processing is associated with heart defects in myotonic dystrophy. Nat Struct Mol Biol 18: 840-845, 2011. 